\title{
Aneurysm of the superficial temporal artery following parotid gland surgery-Case report and review of the literature
}

\author{
Andreas Brandt • Inga-Marie Schaefer • \\ Hans Heino Rustenbeck • Christoph Matthias • \\ Rainer Laskawi
}

Received: 19 September 2012 / Accepted: 20 December 2012 /Published online: 11 January 2013

(C) The Author(s) 2013. This article is published with open access at Springerlink.com

\begin{abstract}
Possible complications of parotid gland surgery are numerous, just as the reasons for aneurysms of the superficial temporal artery. The occurrence of such an aneurysm as a consequence of parotidectomy, however, has so far only been published once. Here, we report of an aneurysm of the superficial temporal artery following parotid gland surgery. It presented as a pulsating mass in the preauricular region. Combining clinical examination, duplex ultrasound, and CT scan, the diagnosis was readily established and treated with a circumscribed revision at low risk and without complications.
\end{abstract}

Keywords Parotidectomy · Aneurysm · Superficial temporal artery $\cdot$ Complication

\section{Introduction}

To treat benign or malignant tumors of the parotid gland, generally a parotidectomy is performed. Despite sophisticated surgical techniques, the occurrence of complications is not uncommon. Their incidences depend on the underlying disease and thereby on the extent and the severity of the surgical

\footnotetext{
A. Brandt $(\varangle) \cdot$ C. Matthias $\cdot$ R. Laskawi

Department of Otolaryngology, University Medical Center

Göttingen, Robert-Koch-Straße 40,

37077 Göttingen, Germany

e-mail: andreas.brandt@med.uni-goettingen.de
}

\section{I.-M. Schaefer}

Department of Pathology, University Medical Center Göttingen, Göttingen, Germany

H. H. Rustenbeck

Department of Neuroradiology, University Medical Center

Göttingen, Göttingen, Germany procedure. Common complications include paralysis or palsy of the facial nerve, Frey's syndrome, parotid fistula, hematoma, infection, and hypoesthesia (for review, see MarcheseRagona et al.) [1]. The development of an arterial aneurysm is, however, not typically seen after surgical treatment, with the exception of vascular catheterization and vascular surgery. In $75 \%$ of cases, the aneurysm of the superficial temporal artery results from blunt trauma [2] and is the most common ( $85 \%$ of cases) traumatic aneurysm in the head and neck region [3]. A recent review reported a total of 166 superficial temporal artery aneurysms published from 1861 to December 2010 [4]. Preferential sites of occurrence are the zygomatic arch and above the linea temporalis, where the vessel is exposed to higher mechanical stress due to the close vicinity to the bone. Clinically, a slowly growing pulsating tumor can be found within a few weeks to month after the trauma. For the diagnosis, duplex ultrasound, CT, and MRI as well as angiography can be used. The differential diagnosis includes benign and malignant neoplasms, infection, abscess formation, and arteriovenous fistula of the facial nerve. The aneurysm can be treated by compression, by surgery, by embolization via vascular intervention catheter, or by direct injection of thrombin $[4,5]$. In 2010, Bernstein et al. were the first to publish the observation of an aneurysm of the superficial temporal artery as a complication of parotid surgery. In this report, a pseudoaneurysm had developed in a 61-year-old male patient 4 weeks after superficial parotidectomy, which was treated by excision [6].

\section{Case report}

A 46-year-old male underwent surgery of the left parotid gland due to cystadenolymphoma. After a postoperative interval of 6 weeks free of complications, a pulsating tumor of the preauricular region had developed. Complaints included 

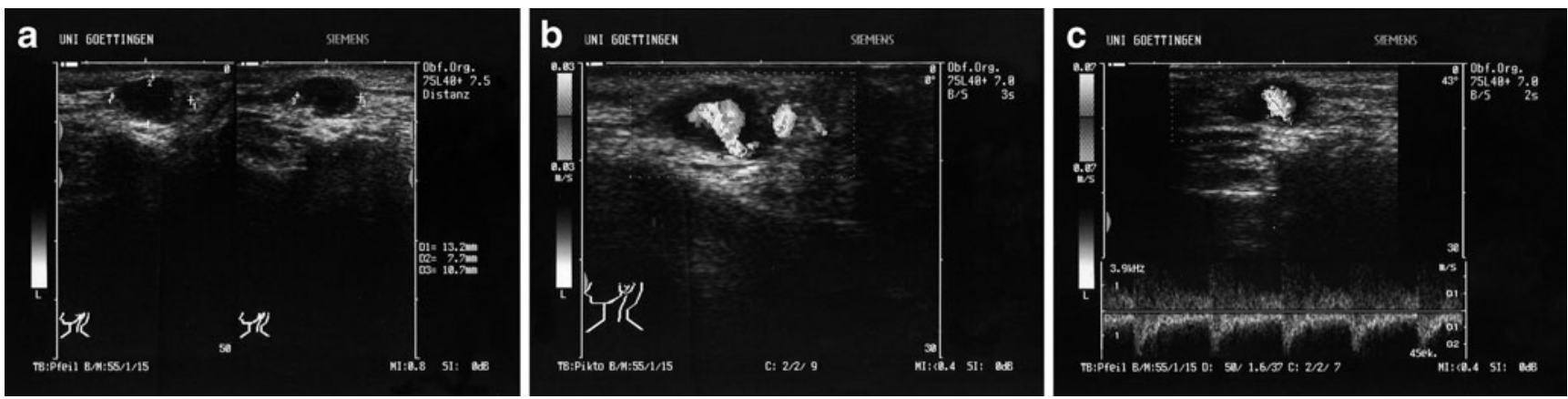

Fig. 1 a Two-dimensional B-mode ultrasound images showing the size of the aneurysm measured to be approximately $13 \times 8 \times 11 \mathrm{~mm}$. b, $\mathbf{c}$ Duplex and Doppler scan visualizing the pulsating blood flow of the aneurysm
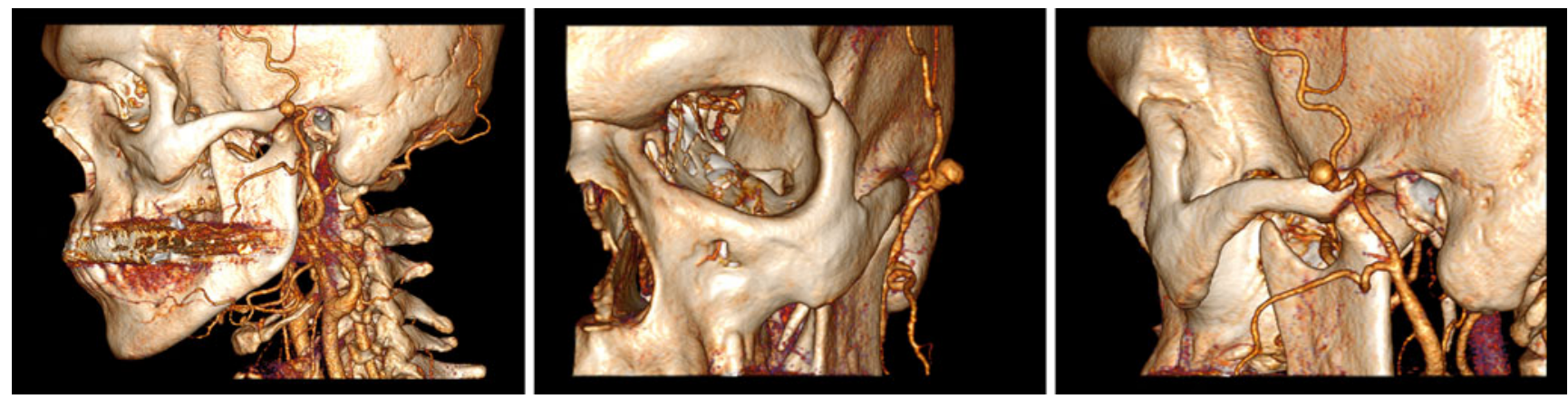

Fig. 2 3-D reconstruction of contrast enhanced spiral CT scan of the head showing the aneurysm located in the left preauricular region. The perfused part of the aneurysm measures approximately $6 \times 7 \mathrm{~mm}$ in size and is fed by the relatively strong superficial temporal artery

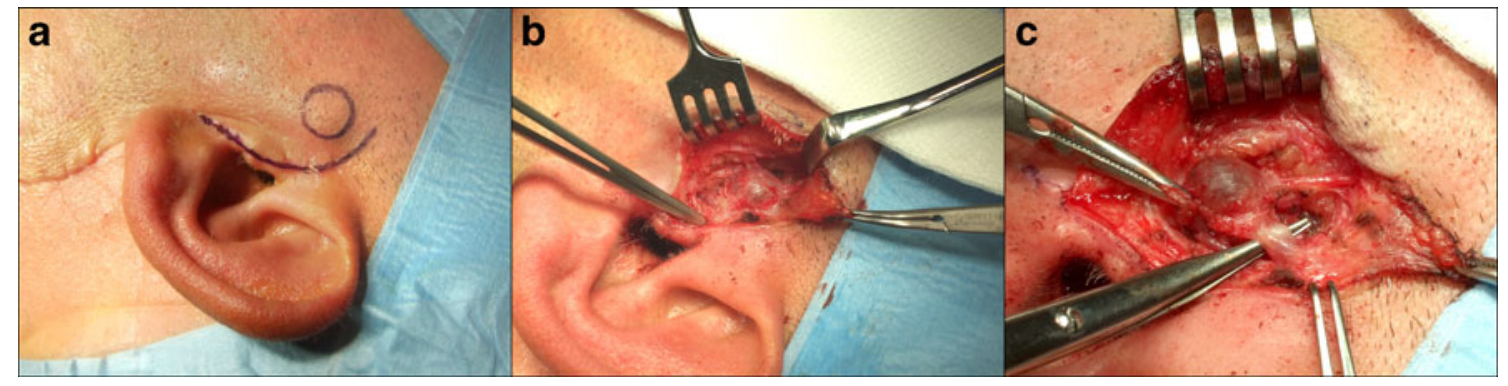

Fig. 3 The aneurysm was removed by revision surgery of the upper parotid region. a Location of aneurysm (circle) and intended incision (line) have been marked. b Operative view before mobilization. c
Higher magnification of operative view before removal of the aneurysm with the draining vessel being lifted

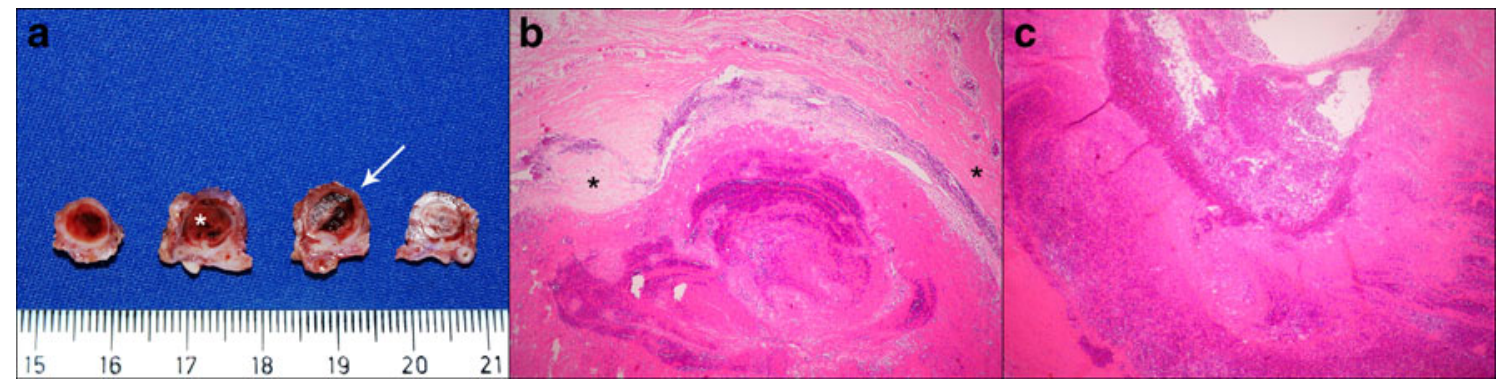

Fig. 4 a On the cut surface, the resected specimen showed an aneurysmatic dilation (asterisk) of the superficial temporal artery (arrow) which was almost completely filled-out with thrombotic material. b, c On microscopic examination, the thrombus was attached to the intima (asterisk) and comprised layered erythrocytes and fibrin, leaving only a small lumen (hematoxylin-eosin, $\times 20, \times 40$ ) 
a feeling of pressure, sharp pain, and recurrent swelling. The lesion was found to be an aneurysm of the superficial temporal artery by using duplex ultrasound (Fig. 1) and a contrast enhanced spiral CT scan of the head (Fig. 2). The aneurysm was treated by circumscribed revision surgery of the upper parotid region under general anesthesia. Afferent and efferent vessels were identified and ligated. The aneurysm was then excised to prevent any further discomfort (Fig. 3). After surgery, no further complications, particularly no facial nerve paralysis, were observed, and the patient was dismissed on the second day after surgery. The following histopathological analysis revealed, as expected, a false aneurysm of approximately $10 \mathrm{~mm}$ in diameter. It was almost completely filled-out by a thrombus consisting of layered fibrin and erythrocytes (Fig. 4).

\section{Discussion}

A tumorous swelling in the preauricular region following parotid surgery can occur for different reasons. Especially hematoma, seroma, abscess, a local recurrence of the tumor, or neuroma of the great auricular nerve have to be considered [1]. In the case presented here, it was the clinical presentation that led to the diagnosis of an aneurysm. Due to the pulsation of the tumor, a process other than vascular could be excluded. However, in case of extensive thrombus formation within the aneurysm, the pulsating character of the swelling can be difficult to detect or completely lacking. Hence, in addition to the clinical examination, at least duplex ultrasound investigation should be performed to clarify the nature of the observed complication.

Treatment of such a pseudoaneurysm is recommended to prevent expansion harboring the risk of bleeding as well as persisting complaints. We preferred a surgical revision to embolization in order to gain local control and at the same time minimizing risk. The revision surgery was performed under general anesthesia to minimize the risk of damage to the facial nerve.

Since the most common reason for aneurysms of the superficial temporal artery is blunt trauma, one might assume a sharp injury to the wall of the vessel during surgery as the underlying event in this case. In 2010, Bernstein et al. [6] reported a similar case of an aneurysm of the superficial temporal artery occurring 4 weeks after superficial parotidectomy, also suggesting an intraoperative trauma to the vessel. Although in both so far observed and published cases the aneurysms emerged after superficial parotidectomy, we would expect the likelihood of injury to the superficial temporal artery to be higher in surgery of cranial inner lobe tumors because of the greater probability to come in close contact with the vessel.

In conclusion, the occurrence of a false aneurysm of the superficial temporal artery following parotid surgery is an extremely rare event. It can be easily detected using noninvasive diagnostic methods. Treatment should be carried out either by revision surgery or by embolization of the artery.

Open Access This article is distributed under the terms of the Creative Commons Attribution License which permits any use, distribution, and reproduction in any medium, provided the original author(s) and the source are credited.

\section{References}

1. Marchese-Ragona R, De Filippis C, Marioni G, Staffieri A (2005) Treatment of complications of parotid gland surgery. Acta Otorhinolaryngol Ital 25(3):174-8

2. Evans CC, Larson MJ, Eichhorn PJ, Taylor RS (2003) Traumatic pseudoaneurysm of the superficial temporal artery: two cases and review of the literature. J Am Acad Dermatol 49(5 Suppl):S286-8

3. Conner WC III, Rohrich RJ, Pollock RA (1998) Traumatic aneurysms of the face and temple: a patient report and literature review. Ann Plast Surg 41(3):321-6

4. van Uden DJ, Truijers M, Schipper EE, Zeebregts CJ, Reijnen MM (2012) Superficial temporal artery aneurysm: Diagnosis and treatment options. Head Neck. doi:10.1002/hed.21963

5. Isaacson G, Kochan PS, Kochan JP (2004) Pseudoaneurysms of the superficial temporal artery: treatment options. Laryngoscope 114 (6): $1000-4$

6. Bernstein JM, Bury RW, Nigam A (2010) Superficial temporal artery pseudoaneurysm: first reported case after parotid surgery. J Laryngol Otol 124(4):441-2 